\title{
Global analyses of TetR family transcriptional regulators in mycobacteria indicates conservation across species and diversity in regulated functions
}

\author{
Ricardo J. C. Balhana ${ }^{1,2}$, Ashima Singla ${ }^{1,3}$, Mahmudul Hasan Sikder ${ }^{1,4}$, Mike Withers $^{1}$ and Sharon L. Kendall ${ }^{1 *}$
}

\begin{abstract}
Background: Mycobacteria inhabit diverse niches and display high metabolic versatility. They can colonise both humans and animals and are also able to survive in the environment. In order to succeed, response to environmental cues via transcriptional regulation is required. In this study we focused on the TetR family of transcriptional regulators (TFTRs) in mycobacteria.

Results: We used InterPro to classify the entire complement of transcriptional regulators in 10 mycobacterial species and these analyses showed that TFTRs are the most abundant family of regulators in all species. We identified those TFTRs that are conserved across all species analysed and those that are unique to the pathogens included in the analysis. We examined genomic contexts of 663 of the conserved TFTRs and observed that the majority of TFTRs are separated by $200 \mathrm{bp}$ or less from divergently oriented genes. Analyses of divergent genes indicated that the TFTRs control diverse biochemical functions not limited to efflux pumps. TFTRs typically bind to palindromic motifs and we identified 11 highly significant novel motifs in the upstream regions of divergently oriented TFTRs. The C-terminal ligand binding domain from the TFTR complement in M. tuberculosis showed great diversity in amino acid sequence but with an overall architecture common to other TFTRs.

Conclusion: This study suggests that mycobacteria depend on TFTRs for the transcriptional control of a number of metabolic functions yet the physiological role of the majority of these regulators remain unknown.
\end{abstract}

Keywords: TetR, Mycobacteria, Tuberculosis, Motif analysis, Gene regulation, Conservation analysis

\section{Background}

TetR family transcriptional regulators (TFTRs) are common one-component prokaryotic signal transduction systems. This family of regulators contain a conserved helix turn helix $(\mathrm{HTH})$ motif at the N-terminal DNAbinding end of the protein and a ligand binding pocket at the C-terminal end. TFTRs are often repressors and bind to DNA to prevent transcription in the absence of a ligand. The binding of an effector molecule at the Cterminal pocket causes structural changes in the protein resulting in the release of the regulator from the DNA.

\footnotetext{
* Correspondence: skendall@rvc.ac.uk

'Department of Pathology and Pathogen Biology, The Royal Veterinary College, Royal College street, Camden, London NW1 OTU, UK

Full list of author information is available at the end of the article
}

TFTRs are present in a large number of bacterial genomes with soil dwelling bacteria encoding the highest numbers [1]. The sequences for more than 200,000 TFTRs are available in public databases and structures for almost 200 have been solved. The paradigm was first described in Escherichia coli and TetR, the founding member of the family, is a repressor that controls the expression of a divergently oriented efflux pump that transports tetracycline out of the cell [2]. Tetracycline binds to the C-terminal ligand pocket of the E. coli TetR to alleviate repression of the pump. In general, TFTRs are best known to bind small molecule ligands to control divergently oriented efflux pumps and, in addition to $E$. coli TetR, there are several good model systems including Staphylococcus aureus QacR [3].

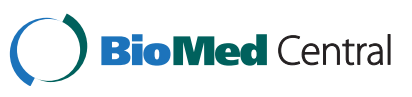

(c) 2015 Balhana et al. This is an Open Access article distributed under the terms of the Creative Commons Attribution License (http://creativecommons.org/licenses/by/4.0), which permits unrestricted use, distribution, and reproduction in any medium, provided the original work is properly credited. The Creative Commons Public Domain Dedication waiver (http:// creativecommons.org/publicdomain/zero/1.0/) applies to the data made available in this article, unless otherwise stated. 
Although the control of drug efflux is a much documented role for this family, as more TFTRs are characterised we are beginning to appreciate that efflux is just one of the diverse functions controlled by this family. The range of TFTR controlled functions include: carbon metabolism, nitrogen metabolism, co-factor metabolism, cell to cell signalling and cell division [1]. TFTRs that do not conform to the paradigm and act as activators [4-6], serve as global regulators [7], interact with peptide ligands [8] and even regulate enzyme activity post-translationally [9] are being described. These observations clearly suggest that there is still much to be learned about this ubiquitous family.

In this paper we use computational analyses to characterise mycobacterial TFTRs. Mycobacteria comprise some of the most important bacterial pathogens including the main causative agents of human and veterinary tuberculosis (Mycobacterium tuberculosis and Mycobacterium bovis, respectively). The exposure to a series of different conditions inside the host, most of which are hostile, and the presence of actively growing and dormant stages imply a key role for the regulation of gene expression in the success of these pathogens. In $M$. tuberculosis, TFTRs are involved in controlling the expression of genes required for carbon utilisation, $k s t R$, kstR2 and mce3R [10-12], branched chain amino acid catabolism, bkaR [13] and antibiotic resistance, Rv3066, ethR $[14,15]$.

We show that TFTRs are the most abundant family of $\mathrm{HTH}$ regulators in mycobacteria and as such the majority remain uncharacterised. We identify all the TFTRs in 10 mycobacterial species and assess the conservation of these genes across the mycobacteria. We define a set of TFTRs that are conserved across all species and those that are unique in those species that cause tuberculosis. It has been shown that genomic context is a reliable tool for predicting the genes regulated by TFTRs [16] and so we use context to predict the functions controlled by a sub-set of mycobacterial TFTRs. TFTRs typically bind to palindromic operators, and we use MEME [17] to identify regulatory motifs in the intergenic regions of the divergently oriented conserved mycobacterial TFTRs.

\section{Results and discussion}

\section{TFTRs are the most abundant type of HTH DNA binding} proteins in mycobacterial genomes

The majority of HTH-containing DNA binding proteins are sub-divided into families based on the structure and spatial arrangement of the helices [18]. InterPro [19] was used to identify the total complement of HTH DNAbinding proteins across 10 mycobacterial genomes (see Methods) and to classify the mycobacterial HTH proteins into their different families. The results, alongside the number of ORFs of each of the species are given in Table 1.

We identified a total of 2338 HTH DNA binding proteins across the 10 mycobacterial genomes. Of these 2338, 906 are TFTRs. For the mycobacterial species analysed, the number of $\mathrm{HTH}$ DNA-binding proteins increases with increasing number of ORFs. In general the soil-dwelling species such as $M$. gilvum and $M$. smegmatis have a larger number of ORFs and so might be expected to contain a larger number of HTH DNA binding proteins. If we compare $M$. gilvum with $M$. marinum, two mycobacteria with similar genome size but one soil dwelling and one adapted for survival in fish and amphibians, we see a reduction in the number of HTH DNA-binding proteins in the host adapted species (272 for M. marinum compared to 328 for M. gilvum) indicative of a reduction in diversity of the conditions within the intra-cellular environment.

TFTRs make up 26-48 \% of the HTH DNA-binding capacity in all species (Table 1, column 3 in brackets). In order to determine if the TFTRs were the most abundant type of HTH DNA-binding protein, the entire HTH complement across the 10 mycobacterial species was classified into family using InterPro. A complete list of genes belonging to each HTH family in all 10 genomes is given in

Table 1 The total number of HTH proteins (including TFTRs) in mycobacterial genomes

\begin{tabular}{|c|c|c|c|c|}
\hline Organism & Number of HTH DNA binding proteins & Number of TFTRs (\% of HTH) & Gene number & TFTRs as a $\%$ of ORFs \\
\hline Mycobacterium tuberculosis & 161 & $52(32 \%)$ & 3999 & 1.3 \\
\hline Mycobacterium bovis & 161 & $51(32 \%)$ & 3920 & 1.3 \\
\hline Mycobacterium bovis $B C G$ & 160 & $51(32 \%)$ & 3952 & 1.3 \\
\hline Mycobacterium avium & 274 & $131(48 \%)$ & 4910 & 2.7 \\
\hline Mycobacterium avium paratuberculosis & 228 & $110(48 \%)$ & 4350 & 2.5 \\
\hline Mycobacterium marinum & 272 & $124(46 \%)$ & 5424 & 2.3 \\
\hline Mycobacterium ulcerans & 201 & $88(44 \%)$ & 4160 & 2.1 \\
\hline Mycobacterium leprae & 39 & $10(26 \%)$ & 1605 & 0.6 \\
\hline Mycobacterium gilvum & 328 & 129 (39\%) & 5531 & 2.4 \\
\hline Mycobacterium smegmatis & 514 & $160(31 \%)$ & 6716 & 2.4 \\
\hline
\end{tabular}


Additional file 1: Table S1. The numbers of genes in each HTH family in 10 mycobacterial species are shown in Fig. 1. Within the HTH superclass, TFTRs are by far the most represented in all mycobacterial genomes. The next best represented HTH classes are GntR, enriched in $M$. smegmatis with 62 assignments but with a small number of representatives in the pathogenic mycobacteria, and OmpR - 14-15 members in all mycobacteria excluding M. leprae.

M. leprae has a drastically reduced genome and so a reduction in the number of TFTRs is expected. In order to determine whether the level of reduction in TFTRs was proportional to genome size we calculated the numbers of TFTRs as a percentage of open reading frames (Table 1, column 5). Interestingly, the percentage of TFTRs in the $M$. leprae was only $0.6 \%$, far less than the other mycobacteria possibly reflecting a disproportionate loss of this family in this species.

It is difficult to say whether mycobacterial genomes are enriched for TetR regulators from this analysis but by way of comparison, E. coli encodes 261 DNA-binding transcription factors in its $4.6 \mathrm{Mbp}$ genome, of which only $5 \%$ are TFTRs [1]. Staphylococcus pyogenes, another intra-cellular Gram positive pathogen, encodes approximately 81 DNA-binding factors, as part of its
$1.85 \mathrm{Mbp}$ genome, of which $\sim 5 \%$ are TFTRs. Soil dwelling bacteria are known to have a large number of TFTRs and so the large numbers in the pathogenic mycobacteria may be a reflection of their evolution from a soil dwelling ancestor [1].

\section{Conservation of TFTRs among the mycobacteria indicates a role in survival for both the environmental and pathogenic species}

The advantage of assessing conservation at the genus level is that it might help to distinguish those TFTRs that are involved in shared processes from those that are required for the more adaptive functions. This is particularly important for mycobacteria where different species have different hosts in addition to environmental representatives. Conservation was assessed as described in the materials and methods. The results are given in Additional file 2: Table S2.

When $M$. leprae is included in the analysis, there are five TFTRs that are conserved across all mycobacteria analysed. These are shaded in blue in the Additional file 2: Table S2 (Rv0238, Rv0472c, Rv3050c, Rv3208 and Rv3855 $(e t h R))$. The conservation of these regulators across all mycobacterial genomes, including the drastically reduced $M$. leprae genome suggests that the functions of these

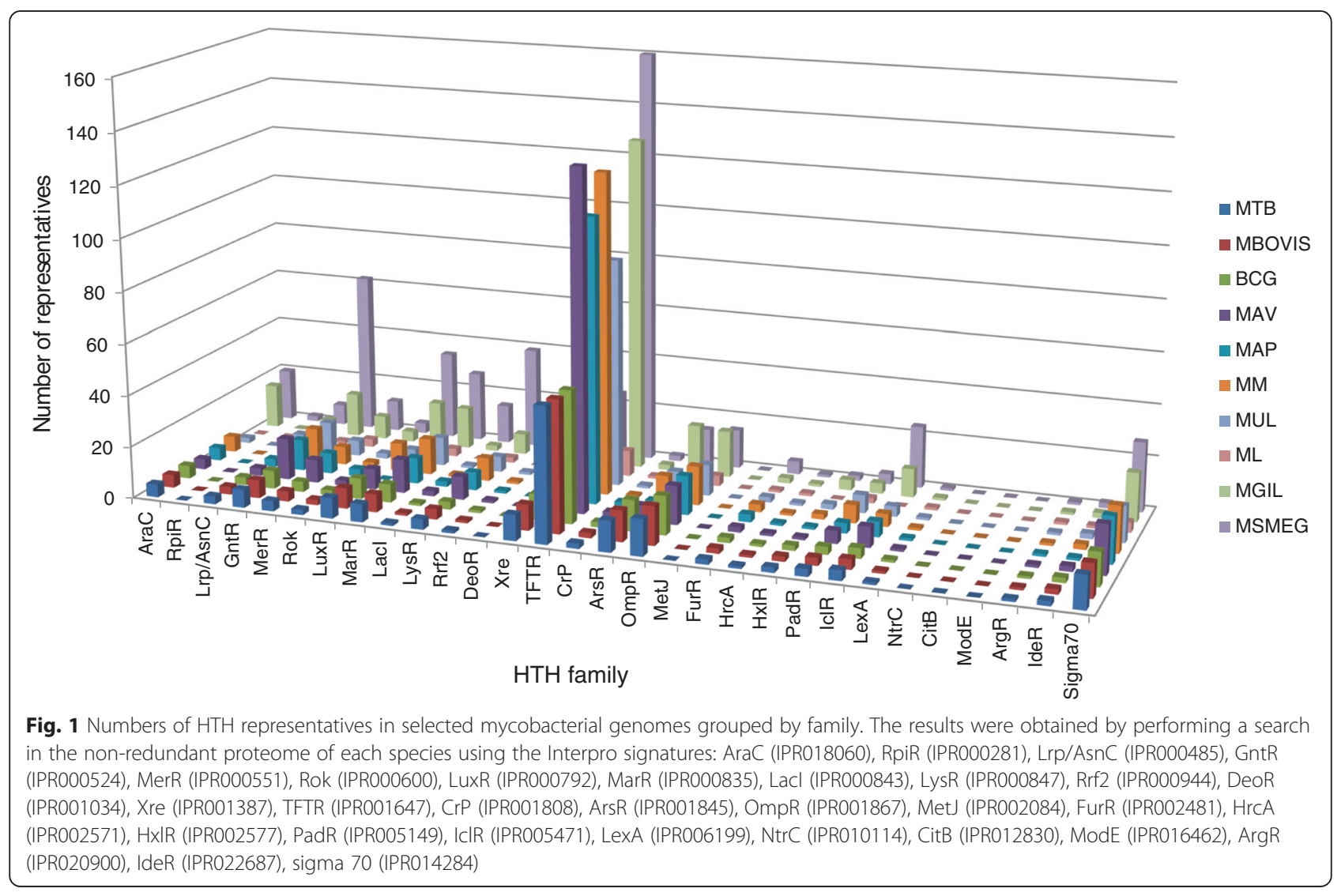


regulators are required for survival in both host adapted and environmental niches. The M. leprae gene ML2457 is divergently oriented to a pseudogene and may not have a physiological role in this species. This group of regulators include $e t h R$, a TFTR involved in antibiotic resistance that represses genes required for the activation of the antibiotic ethionamide. Mutations in this regulator cause resistance [15]. Its conservation in M. smegmatis and M. gilvum suggests that it might be useful in this species as a defence mechanism against antibiotic producers in the soil in the battle for resources.

Given that M. leprae has a much reduced genome and our previous analysis suggested a disproportionate loss of TFTRs we re-assessed conservation across mycobacterial genomes but this time excluded M. leprae. Those TFTRs that are conserved across all mycobacteria (excluding M. leprae) are shaded in green in Additional file 2: Table S2. The TFTRs present in M. tuberculosis are, in general well conserved with 22 of the 52 regulators having orthologs in all species included in the analysis. This group of regulators include $k s t R$ (Rv3574) and kstR2 $(R v 3557 c)$, involved in cholesterol catabolism $[10,11,20]$. Their conservation in both pathogenic and environmental species suggests sterols are likely to be encountered in the environment (phytosterols and ergosterols) as well as in the host (host cholesterol). The conservation of the KstR regulators in M. avium subspecies paratuberculosis suggests that cholesterol catabolism is also important for this intestinal pathogen. This is supported by the recent observation that cholesterol is a carbon source for $M$. avium subspecies paratuberculosis in the bovine intestine [21].

\section{Conservation analysis identifies those TFTRs that are only present in the pathogenic representatives}

In order to identify those TFTRs that might be uniquely involved in pathogenic processes (i.e. conserved in the pathogens but not conserved in the environmental species) we identified those TFTRs that were missing from both $M$. smegmatis and M. gilvum but present in the pathogenic species (Additional file 2: Table S2).

Only one regulator (Rv0078, shaded purple in Additional file 2: Table S2), was present in all pathogens, including $M$. leprae. However, the ortholog in M. leprae (ML2677) is divergently oriented to a pseudogene and so it is possible that it does not have a physiological role in M. leprae. Excluding the disproportionately reduced $M$. leprae from the analysis, three TFTRs (Rv0653c, Rv1167c and Rv1556) are conserved in the pathogenic species only and these are shaded in orange in the Additional file 2: Table S2. These candidates might control functions uniquely important for survival in the host.

Six genes were uniquely found in the species that cause tuberculosis (Rv0302, Rv0328, Rv0330c, Rv1534, Rv2160A and $R v 3160 c$ ). These genes are shaded in red in Additional file 2: Table S2. With the exception of Rv3160c and $R v 2160 A$, we currently do not have any experimental evidence of the functions that these six TFTRs might control. There is a frame shift mutation in $R v 2160 A$ in $M$. tuberculosis that makes it non-functional in this species. $R v 2160 A$ is situated on a likely operon with upstream and downstream genes $R v 2159 c$ and $R v 2161 c$, respectively. These flanking genes show higher expression in $M$. tuberculosis and differential expression might have an impact on host preference [22]. $R v 2159 c$ is annotated as an alkyl hydro peroxidase, whereas $R v 2161 c$ is a conserved hypothetical protein. Their role in the physiology of the bacterium is unknown. Rv3160c and the neighbouring genes Rv3161c (a dioxygenase) and $R v 3162 c$ (a membrane protein) are induced upon exposure to antibiotics but the precise physiological functions of these genes remain unknown [23].

\section{The TFTR regulator $R v 1255 c$ is present in $M$. tuberculosis but missing from $M$. bovis and the vaccine strain $M$. bovis BCG Pasteur}

The sequence of the M. bovis and M. tuberculosis genomes are $99.95 \%$ similar and it has often been hypothesised that the widely different host preference exhibited by these species is a reflection of changes in gene expression rather than content. Aside from $R v 1255 c$, the complement of TFTRs in M. bovis and M. tuberculosis is identical.

$R v 1255$ c lies in the RD10 region which is part of a series of deletions that occurred in the "ancestral" TbD1 +- species in the Mycobacterium africanum $\rightarrow$ Mycobacterium microti $\rightarrow$ M. bovis lineage. The RD10 deletion is present in strains that show wide host diversities and geography such as humans in Africa, voles in the UK, seals in Argentina, goats in Spain, and cattle and badgers in the UK $[24,25]$. This regulator is on a putative two gene operon with the cytochrome $\mathrm{p} 450$ cyp130 (Rv1256c), also within the RD10 region. Studies of the function and regulation of CYP130 in the "modern" - TbD1- strains of human adapted $M$. tuberculosis might allow us to gain additional knowledge of some of the biochemical differences between "modern" M. tuberculosis and "ancestral" and animal adapted species.

Similarly there are deletions in TFTRs in other strains of $M$. bovis BCG that might influence the efficacy of the vaccine. $R v 3405 c$ is in the RD16 region deleted from $M$. bovis BCG Moreau but a link between this deletion and vaccine efficacy is unknown [26].

\section{Most mycobacterial TFTR regulators are divergently oriented to an adjacent gene}

It has been recently reported by Ahn et al., that examination of the genome context of TFTRs can be a useful tool for the prediction of the genes they regulate [16]. 
This study, which focused on Streptomyces, showed that TFTRs that are divergently oriented to their neighbouring genes and separated by 200 bp or less can be reliably predicted to control the neighbouring gene. This analysis showed that the functions of the neighbouring gene(s) were more diverse than just drug efflux.

In order to examine the situation in mycobacteria, we analysed 663 TFTRs from M. tuberculosis, M. avium paratuberculosis, M. marinum, M. ulcerans, M. gilvum and $M$. smegmatis, for orientation, length of intergenic region and function of adjacent genes. The regulators were classified into groups $(\mathbf{A}-\mathbf{C})$ according to the criteria laid down by Ahn et al. (A) divergent orientation with neighbour, (B) likely to be co-transcribed with upstream or downstream gene as they are in the same orientation and the intergenic DNA separating them is $\leq 35 \mathrm{bp}$, and $(\mathbf{C})$ show neither $(\mathbf{A})$ or $(\mathbf{B})$. The results are shown in Fig. 2.

In all six species approximately $60 \%$ of the TFTRs are divergently oriented with their neighbour and this is similar to the figure reported by Ahn et al., for Streptomyces species. The next most favoured arrangement is co-transcription with neighbouring genes followed by an ambiguous arrangement.
For those that are divergently transcribed, the majority of regulators are separated from their divergent partners by 200 bp or less (Fig. 3). So, for M. tuberculosis 25 out of the 33 divergently oriented genes are separated by $200 \mathrm{bp}$ or less $(76 \%)$ and such high frequencies are also observed in the rest of the mycobacteria (53/64 for $M$. avium paratuberculosis (83 \%), 58/77 for $M$. marinum (75 \%), 34/51 for $M$. ulcerans (67 \%), 74/87 for $M$. gilvum (85 \%) and 96/110 for M. smegmatis (87 \%). These analyses suggest that the majority of the divergently oriented TFTRs can be predicted to regulate the adjacent gene.

\section{Functional analysis of divergently oriented adjacent genes reveals that TFTRs control a diverse range of metabolic functions not limited to efflux}

We examined the functions of the genes divergent to the TFTRs in the six mycobacterial genomes in order to determine the possible functions regulated. We only included those genes that were separated from their divergent TFTRs by $200 \mathrm{bp}$ or less. 340 genes from four different genomes (M. tuberculosis, M. avium paratuberculosis, M. marinum M. ulcerans, M. gilvum and $M$.

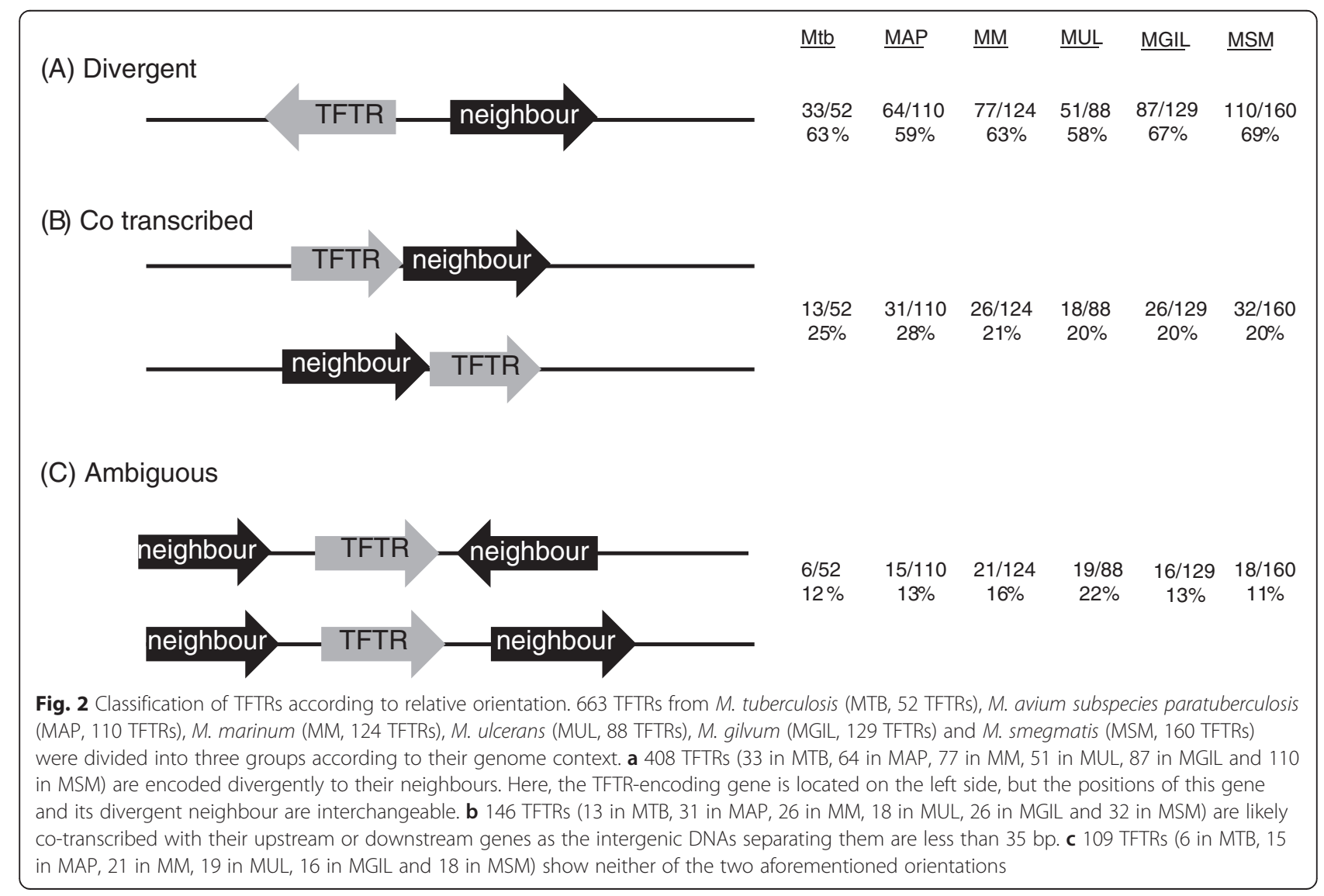




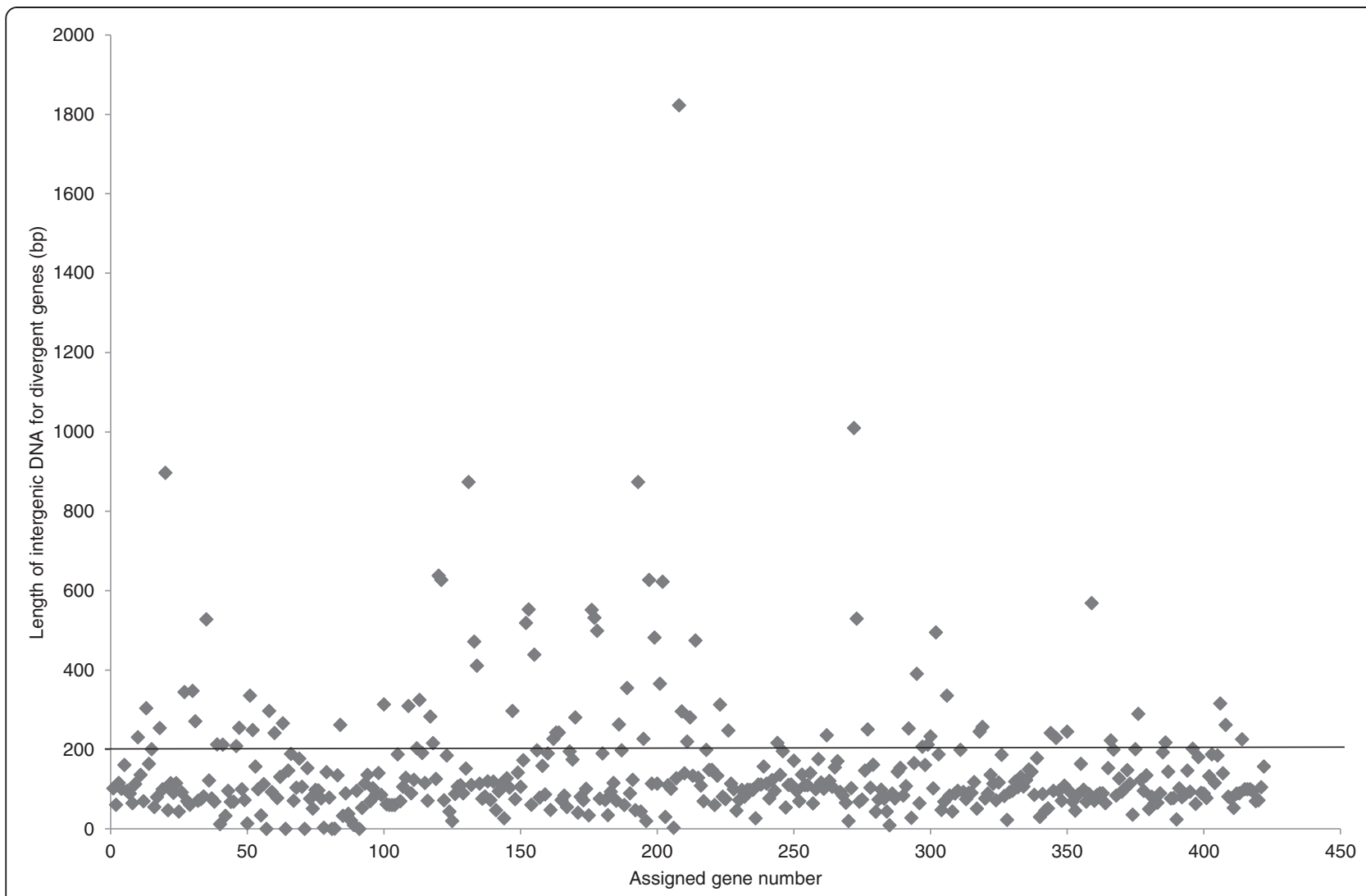

Fig. 3 Lengths of the intergenic regions of the divergently oriented mycobacterial TFTR regulators. The intergenic regions from the 422 divergently oriented regulators from M. tuberculosis (Mtb), M. avium paratuberculosis (MAP), M. marinum (MM), M. ulcerans (MUL), M. gilvum (MGIL) and M. smegmatis (MSM) were analysed for length. Each dot represents an intergenic region and the length is given on the $y$-axis. Each of the genes were assigned a number e.g. 1-33 for MTB, 34-97 for MAP, 98-174 for MM, 175-225 for MUL, 226-312 for MGIL and 313-422 for MSMEG. The assignation of number was done in gene number order in each organism e.g. $1=$ Rv0067c, $2=$ Rv0078, $3=$ Rv0135c etc. and this is given on the $x$-axis. The line represents a cut-off intergenic region size of $200 \mathrm{bp}$. The graph shows that the majority of divergently oriented genes are separated from their neighbour by $200 \mathrm{bp}$ or less

smegmatis) were analysed in total. The results are shown in Fig. 4.

Fifty-eight percent of the divergently oriented genes are enzymes. The predicted enzymes were sub-divided into Enzyme Commission (EC) number according to the reactions they were predicted to catalyse and by the presence of domains associated with that particular enzyme class. The majority of enzymes $(40 \%)$ are oxidoreductases (EC1) indicating that, in mycobacteria, the majority of TetR regulators control the expression of enzymes involved in energy and cellular metabolism, which may be crucial for metabolic adaptation.

Membrane proteins only account for $10 \%$ of the functions of divergently oriented genes and attempts to further classify these were made using Pfam (http:// pfam.xfam.org/) and Superfamily (http://supfam.cs.bris.ac.uk/SUPERFAMILY/). 22 of the 35 membrane proteins gave either no hits or contain a conserved domain of unknown function (pfam04286). 5 of the membrane proteins belong to the major facilitator superfamily of transporters (cl18950), 2 are PPE family proteins (pfam00823), 1 contains a mycobacterial membrane protein domain (pfam05423), 1 is a membrane bound histidine kinase (pfam00672), 1 is a chloride channel protein (pfam00654), 1 is a sodium decarboxylate symporter family (pfam00375), 1 is an $\mathrm{ABC}$ transporter family (pfam01061) and 1 is an amino acid permease (pfam13906).

These results are in agreement with the findings by Ahn et al. [16], and lend further support to the realisation that TFTRs do not just regulate efflux pumps. Our analyses suggest that TFTRs regulate a diverse range of as yet uncharacterised metabolic functions.

\section{Analysis of the upstream region of divergent TFTRs identifies 11 novel putative binding motifs}

TFTRs typically bind to palindromic operators. The model TetR from $E$. coli binds as a dimer to a 15 bp 


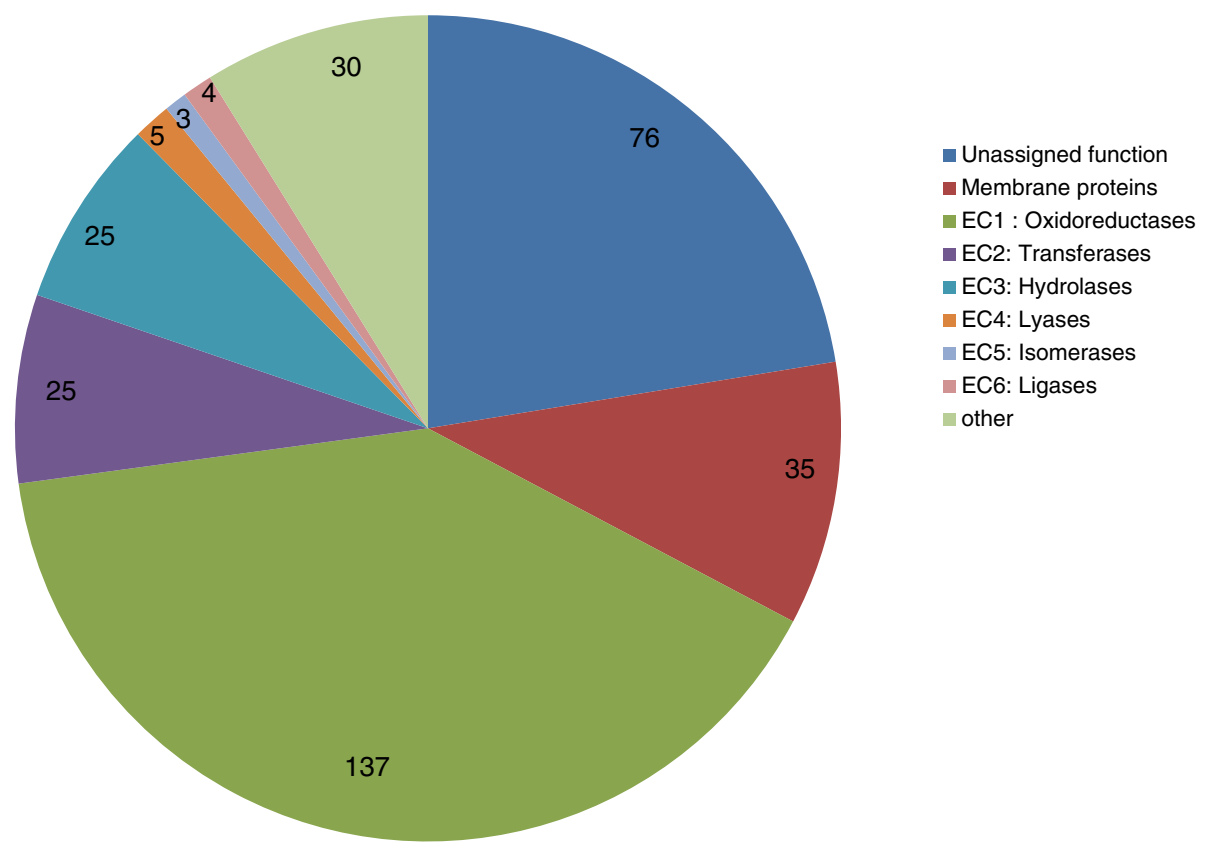

Fig. 4 Functional classification of the products encoded by the divergent neighbouring genes. Genes that were divergently oriented to TFTRs with an intergenic region of 200 bp or less in M. tuberculosis, M. marinum, M. avium paratuberculosis and M. smegmatis were analysed as described in the materials and methods. Gene products that were enzymes were classified according to class (EC 1 to EC 6). Non enzymatic products were classified into membrane proteins, other proteins (e.g. transcriptional regulators), and proteins of unassigned function

palindrome while QacR from S. aureus binds as a tetramer to a 28 bp palindrome [3]. A number of TFTRs from M. tuberculosis (Rv3066, KstR, KstR2, BkaR) also bind to palindromic motifs $[10,11,13,14]$. Motifs for Mce3R and EthR have also been described but these are larger, more complex, in multiple copies and do not conform to the classical structure of a palindromic sequence separated by a small number of bases $[15,27]$.

We used the programmes MEME and MAST to identify regulatory motifs in the intergenic regions for those regulators that were conserved across a number of species and were divergently oriented to the neighbouring gene [17]. A total number of 30 TFTRs were examined in the analysis, including the previously experimentally verified motifs. The results are given in Table 2 .

The experimentally verified motifs show an e-value of $>\mathrm{E}-20$ therefore we classified the motifs into highly significant (e value is $=$ or $>E-20$ ) and less significant $(<\mathrm{E}-20)$. Of the 30 motifs that were analysed, 11 passed the cut-off. These represent a set of probable TetR binding motifs for the regulators listed.

\section{Conservation analysis of the C-terminal domain of TFTRs in M. tuberculosis}

Although the work presented here and elsewhere support the idea that it is straightforward to predict at least one direct target gene for a previously unstudied TFTR, the real challenge is in the determination of the small- molecule ligands that the TFTRs bind to at the Cterminal end. Identification of the ligand is a means by which the biochemical function(s) of the target genes can be elucidated as many of the ligands bound by TFTRs are related to the biochemical functions of the target gene [28].

Previously phylogenetic analysis has been use to subdivide the GntR family of regulators in $M$. tuberculosis into functional clades based on the amino acid sequence similarity of their effector domain [29]. Additionally, phylogenetic approaches have been used to make general functional predictions for transcription factors for the AraC family [30]. Potentially a similar analysis could be applied to the $\mathrm{C}$-terminal ligand binding domain of TFTRs to sub-divide the family into groups. Previously larger pan-genomic studies have grouped TFTRs based on amino acid sequence including TFTRs with known ligands and used this information to predict a ligand for a TFTR from Streptomyces, a prediction that was experimentally verified [28].

We aligned the C-terminus of all TFTRs in M. tuberculosis and attempted to establish phylogenetic groupings using widely-employed methodologies such as parsimony, maximum likelihood and neighbour joining. The alignments obtained were poor due to very low sequence similarity, approximately $7 \%$ average identity between amino acid sequences. Conversely, the phylogenetic trees obtained (data not shown) showed overall 
Table 2 Motif analysis of the intergenic regions of conserved divergently oriented TFTRs

\begin{tabular}{lll}
\hline Gene number (name) & Motif & e-value \\
\hline Rv2506 (bkaR) & & $1.20 \mathrm{E}-27$ \\
& &
\end{tabular}

Rv3357c (kstR2)

\section{"TACGA}

4.40E-31

Rv3574 (kstR)

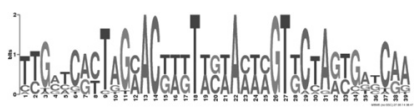

6.90E-36

Rv3405c

Table 2 Motif analysis of the intergenic regions of conserved divergently oriented TFTRs (Continued)

Rv3055

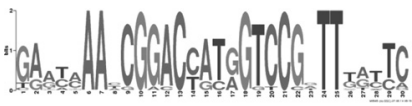

Rv3208

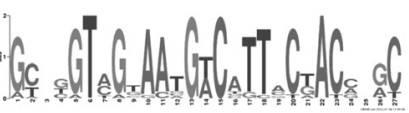

$1.00 \mathrm{E}-25$

Rv3855 (ethR)

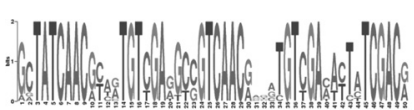

8.90E-34

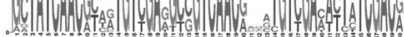

Rv0067c

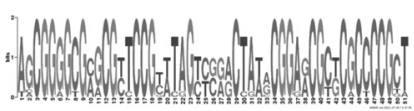

1.10E-29

Rv0144

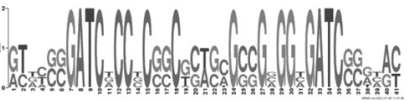

$3.00 \mathrm{E}-16$

:

$2.00 \mathrm{E}-36$

Rv0078

"T TCA

$5.60 \mathrm{E}-31$

Rv0158

Rv0135c

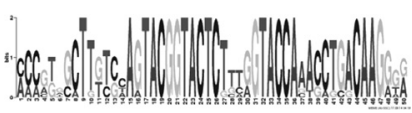

$6.00 \mathrm{E}-80$

Rv0653c

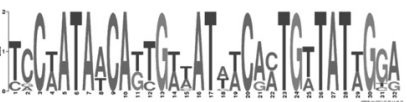

1.10E-18

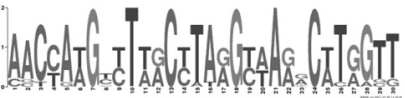

$4.90 \mathrm{E}-13$

Rv0472C

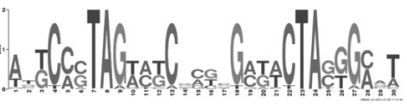

$5.60 \mathrm{E}-16$

$5.60 \mathrm{E}-16$

$1.20 \mathrm{E}-43$

Rv0273c

:

Rv0275c

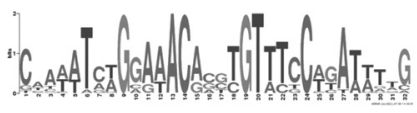

$9.00 \mathrm{E}-27$

Rv0691c

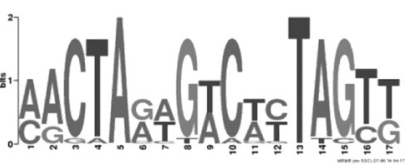

$6.00 \mathrm{E}-17$

*

3.00E-34

Rv0767c 
Table 2 Motif analysis of the intergenic regions of conserved divergently oriented TFTRs (Continued)

Rv0825C

Rv1019

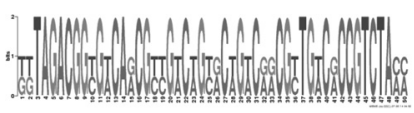

1.90E-17

Rv1776c

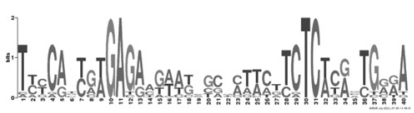

4.00E-16

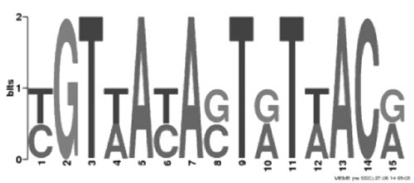

Rv3058c

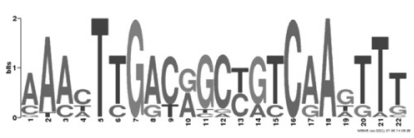

2.00E-12

2.40E-11

$$
\text { termin }
$$
the DNA binding region of TFTRs [18].

\section{Meta-analysis of published essentiality and expression studies triages those for further study and indicates infection relevant physiological functions for a selection of TFTRs}

In order to determine those TFTRs that might have a role during infection in $M$. tuberculosis we performed a meta-analysis of selected published microarray studies to determine those TFTRs that are either essential or show expression changes in infection models or under in vitro conditions that mimic aspects of infection. The results of the analysis is shown in Additional file 4: Table S4.

Twenty-four TFTRs showed expression changes in at least 1 of the experimental conditions while 7 regulators were essential in at least one of the experimental conditions. This analysis helps to prioritise those TFTRs that might be taken forward for further study of the regulatory mechanisms involved in survival of M. tuberculosis.

Four regulators are essential for infection in the mouse model (Rv2912c, Rv3050c, Rv3574 (kstR) and $R v 3855$ (ethR)). The physiological role of $k s t R$ is in the catabolism of cholesterol as a carbon source during infection [10, 20,33], but the physiological role of the other essential TFTRs are unknown. The role of EthR in the control of ethA, an enzyme required for the activation of an anti-tuberculosis therapy ethionamide, is well documented but its physiological role remains unknown [15, 34-36]. Interestingly EthR is also induced under hypoxia and in dendritic cells. This analysis suggests an infection relevant physiological function for this regulator.

\section{Conclusion}

TFTRs are especially frequent in organisms exposed to environmental alterations and stresses, for instance soil bacteria, and, not surprisingly, pathogenic species. 
Mycobacteria are a very versatile genus with species colonising very different environments, from soil dwelling saprophytic organisms, like M. smegmatis and M. gilvum to obligate human pathogens such as $M$. tuberculosis and $M$. leprae and also organisms that can coexist in both a parasitic and a free-living lifestyles such as $M$. marinum and M.ulcerans. To add to this inter-species versatility, the niche and lifestyle of each mycobacterial species can also be quite diverse, for example, $M$. tuberculosis has the ability to cause both life-threatening pulmonary tuberculosis and also clinically latent infections, living intra-cellularly as well as extra-cellularly and capable of infecting extrapulmonary tissues. Such flexibility can only be achieved through changes in genetic expression, which is mostly mediated by transcriptional regulators.

In this work we have shown that the TFTRs are the most abundant family of transcriptional regulators with 906 TFTRs across the 10 species examined. Enrichment with such high numbers of TFTRs in mycobacterial genomes may be because TFTRs tend to control small regulons of neighbouring genes. Our data also suggests that mycobacterial TFTRs regulate divergent functions, including but extending beyond, efflux pumps. In fact, most mycobacterial TFTRs seem to be associated with enzymes which may reflect the metabolic plasticity in these species. Operator motif identification in mycobacteria is still in the early stages with motifs being identified for only a few transcriptional regulators [37]. We have identified 11 putative novel motifs for the TFTRs and these represent a set of sequences for testing. Only a few mycobacterial TFTRs have been well characterised to date, the importance of these in pathogenesis in $M$. tuberculosis or antibiotic resistance signifies that that further research into the uncharacterised TFTRs is necessary.

\section{Methods \\ Identification and classification of the HTH DNA binding proteins in mycobacteria}

The genome sequences of Mycobacterium leprae (NC_ 002677), Mycobacterium bovis AF2122/97 (NC_00 2945), Mycobacterium bovis BCG Pasteur 1173P2 (NC_ 008769), M. tuberculosis H37Rv (NC_000962), Mycobac terium avium subsp. paratuberculosis K-10 (NC_00 2944) Mycobacterium avium (NC_008595), Mycobacterium marinum (NC_010612), Mycobacterium ulcerans (NC_005916), Mycobacterium gilvum (NC_009338) and Mycobacterium smegmatis $m^{2} 155$ (NC_008596) were used in the analysis. These genomes represent those species that are obligate pathogens (M. leprae, $M$. bovis and $M$. tuberculosis), those that are able to cause disease but also survive outside the host ( $M$. avium, $M$. marinum $M$. avium subsp. paratuberculosis and $M$. ulcerans) and those that are purely environmental $(M$. smegmatis and $M$. gilvum). The entire genome sequence from each species was searched using the integrated database Interpro in order to identify $\mathrm{HTH}$ DNA binding proteins and classify them into families.

\section{Conservation analysis of the TFTRs}

Assessment of conservation and identification of orthologs was done using a combination of protein BLAST, using the NCBI web server (http://www.ncbi.nlm.nih.gov/), and TB database [38] available at (http://tbdb.org/). Orthologs were identified by reciprocal protein-protein BLASTS (blastp algorithim). For example the amino acid sequence of a TFTR of one mycobacterium was compared to the entire ref-seq protein complement of the M. tuberculosis genome. The sequence of the most highly similar $M$. tuberculosis protein was used in another protein-protein BLAST against the original mycobacterium. Those genes that identified each other (i.e. reciprocal pairs) were considered potential orthologs. Pairwise identities were much less than 0.01 in each case and were more in the region of 1e-100. Amino acid sequence identities were greater than $50 \%$ in each case over the entire length of the protein. Additionally, we used synteny as auxillary information for the detection or orthology. These were then checked using the TB database.

\section{Identification and functional analysis of adjacent genes}

Genome context of the TFTRs in each genome was viewed using Artemis [39] available from the Web server (http://www.sanger.ac.uk/resources/software/artemis/). TFTRs were placed into their respective categories $(\mathrm{A}-\mathrm{C})$ and analysed in excel. For the divergently oriented genes the lengths of each of the intergenic regions were determined and only those genes that were separated by 200 bp or less were included in the functional analyses. In order to predict the functions of these divergently oriented genes, protein sequences were downloaded and a combination of protein BLAST, the TB database, and conserved domain search (CD) search was used (http://www.ncbi.nlm.nih.gov/Structure/cdd/wrpsb.cgi). Further analysis of membrane proteins was done using TMHMM [40] available at (http://www.cbs.dtu.dk/services/TMHMM/).

\section{Motif identification}

Motif analysis was performed using MEME (http:// meme-suite.org/) [17]. Intergenic regions from genes that were conserved among the mycobacteria and adjacently oriented genes were used in the analysis. This consisted of a group of 30 genes. The sequence of intergenic regions were extracted and uploaded in FASTA format. MEME was set to find palindromic motifs with a minimum width of 6 and a maximum of $50 \mathrm{bp}$. MEME was set to return a maximum of 3 motifs and the most significant motif was tabulated. 


\section{C-terminal domain analyses}

Multiple sequence alignment of TetR proteins was generated with Clustal software [41] available at (http://memesuite.org/). The multiple alignments were either taken directly from the output generated by Clustal or manually improved based on secondary structure information using JPred 3 [32]. JPred 3 was used with the pre-set parameters available at (http://www.compbio.dundee.ac.uk/wwwjpred/). Phylogenetic analyses were carried out using the PHYLIP software package. The SEQBOOT program was used to generate 1000 bootstrapping datasets for phylogeny estimations by parsimony and neighbour joining and 100 for maximum likelihood. Parsimony analysis was performed by running the multiple datasets through PROTPARS and CONSENSE, while neighbour joining analysis was done using PROTDIST, NEIGHBOUR and CONSENSE. Maximum likelihood analysis was carried out by inputting the 100 datasets in the program PROML and then running CONSENSE.

\section{Additional files}

Additional file 1: Table S1. Classification of all the HTH DNA binding proteins across 10 mycobacterial genomes. The first worksheet is the total number of representatives in each class for each genome. Each genome with the list of genes in each class are on separate worksheets. Each column in a worksheet represents a different HTH family with the family name and IPR number given in the column heading.

Additional file 2: Table S2. Conservation analysis of the mycobacteria TFTRs. Conservation was assessed relative to MTB. Genes that are conserved in all species including ML are shaded in blue. Those that are conserved across all mycobacteria with the exception of ML are shaded in green. Those that are conserved in all the pathogens (including ML) but missing from the non-pathogenic strains are shaded in purple and those that are conserved in all the pathogens (excluding $\mathrm{ML}$ ) but missing from the non-pathogenic strains are shaded in orange. Those TFTRs conserved in those species causing tuberculosis are shaded in red.

Additional file 3: Figure S3. Secondary structure alignment of the C-terminus of the 52 TFTR regulators present in M. tuberculosis together with other regulators described previously in the literature. The structures were obtained using Jpred 3 and the each domain highlighted was aligned separately using ClustalX2.

Additional file 4: Table S4. Meta-analysis of microarray data. All 52 TFTRs from M. tuberculosis were examined for gene expression changes in key publications examining changes in gene expression and essentiality in vivo and in vitro under infection relevant conditions.

\section{Abbreviations}

TFTRs: TetR family transcriptional regulators; HTH: Helix-turn-helix; EC: Enzyme commission, MTB, Mycobacterium tuberculosis; MBOVIS: Mycobacterium bovis; BCG: Mycobacterium bovis BCG; MAV: Mycobacterium avium subspecies avium; MAP: Mycobacterium avium subspecies paratuberculosis; MM: Mycobacterium marinum; MUL: Mycobacterium ulcerans; ML: Mycobacterium leprae; MGIL: Mycobacterium gilvum; MSM: Mycobacterium smegmatis.

\section{Competing interests}

The authors declare that they have no competing interests.

\section{Authors' contributions}

RB and SLK conceived of, designed and wrote the manuscript. RB performed the $\mathrm{C}$-terminal domain analysis and wrote programs to retrieve HTH DNA binding domains in the species used in this manuscript. MW performed the motif analyses. SLK, RB, AS and MS performed the context analysis, intergenic length analysis and functional analysis of adjacent genes in the relevant species. All authors read and approved the final manuscript.

\section{Acknowledgments}

RB was in receipt of an RVC PhD scholarship. AS received a travel grant from the Boehringer Ingelheim Fonds (BIF). MS received funding from the Commonwealth Scholarship Commission. MW was funded by the EU MM-TB STREP Grant No. 012187. The project was supported by the Wellcome Trust (Grant 073237). We thank Dr Liam Good for critically reviewing the manuscript.

\section{Author details}

'Department of Pathology and Pathogen Biology, The Royal Veterinary College, Royal College street, Camden, London NW1 OTU, UK. ${ }^{2}$ Department of Microbial and Cellular Sciences, Faculty of Health and Medical Sciences, University of Surrey, Stag Hill, Guildford GU2 7XH, UK. Indian Institute of Technology Kanpur, Kanpur, India. ${ }^{4}$ Department of Pharmacology, Faculty of Veterinary Science, Bangladesh Agricultural University, Mymensingh 2202, Bangladesh.

Received: 24 October 2014 Accepted: 12 June 2015

Published online: 27 June 2015

\section{References}

1. Cuthbertson L, Nodwell JR. The TetR family of regulators. Microbiol Mol Biol Rev. 2013;77(3):440-75.

2. McMurry L, Petrucci Jr RE, Levy SB. Active efflux of tetracycline encoded by four genetically different tetracycline resistance determinants in Escherichia coli. Proc Natl Acad Sci U S A. 1980;77(7):3974-7.

3. Schumacher MA, Miller MC, Grkovic S, Brown MH, Skurray RA, Brennan RG Structural mechanisms of QacR induction and multidrug recognition. Science. 2001:294(5549):2158-63.

4. Alatoom AA, Aburto R, Hamood AN, Colmer-Hamood JA. VceR negatively regulates the $v c e C A B M D R$ efflux operon and positively regulates its own synthesis in Vibrio cholerae 569B. Can J Microbiol. 2007;53(7):888-900.

5. Hirano $S$, Tanaka K, Ohnishi Y, Horinouchi S. Conditionally positive effect of the TetR-family transcriptional regulator AtrA on streptomycin production by Streptomyces griseus. Microbiology. 2008;154(Pt 3):905-14.

6. Pompeani AJ, Irgon JJ, Berger MF, Bulyk ML, Wingreen NS, Bassler BL. The Vibrio harveyi master quorum-sensing regulator, LuxR, a TetR-type protein is both an activator and a repressor: DNA recognition and binding specificity at target promoters. Mol Microbiol. 2008;70(1):76-88.

7. Beckers G, Strosser J, Hildebrandt U, Kalinowski J, Farwick M, Kramer R, et al. Regulation of AmtR-controlled gene expression in Corynebacterium glutamicum: mechanism and characterization of the AmtR regulon. Mol Microbiol. 2005;58(2):580-95.

8. Goeke D, Kaspar D, Stoeckle C, Grubmuller S, Berens C, Klotzsche M, et al. Short peptides act as inducers, anti-inducers and corepressors of Tet repressor. J Mol Biol. 2012;416(1):33-45.

9. Liu L, Huang C, He ZG. A TetR family transcriptional factor directly regulates the expression of a 3-methyladenine DNA glycosylase and physically interacts with the enzyme to stimulate its base excision activity in Mycobacterium bovis BCG. J Biol Chem. 2014;289(13):9065-75.

10. Kendall SL, Burgess P, Balhana R, Withers M, Ten Bokum A, Lott JS, et al. Cholesterol utilization in mycobacteria is controlled by two TetR-type transcriptional regulators: kstR and kstR2. Microbiology. 2010;156(Pt 5):1362-71.

11. Kendall SL, Withers M, Soffair CN, Moreland NJ, Gurcha S, Sidders B, et al. A highly conserved transcriptional repressor controls a large regulon involved in lipid degradation in Mycobacterium smegmatis and Mycobacterium tuberculosis. Mol Microbiol. 2007;65(3):684-99.

12. Santangelo MP, Blanco FC, Bianco MV, Klepp LI, Zabal O, Cataldi AA, et al. Study of the role of Mce3R on the transcription of mce genes of Mycobacterium tuberculosis. BMC Microbiol. 2008;8(1):38.

13. Balhana RJ, Swanston SN, Coade S, Withers M, Sikder MH, Stoker NG, et al. bkaR is a TetR-type repressor that controls an operon associated with branched-chain keto-acid metabolism in Mycobacteria. FEMS Microbiol Lett. 2013;345(2):132-40. 
14. Bolla JR, Do SV, Long F, Dai L, Su CC, Lei HT, et al. Structural and functional analysis of the transcriptional regulator Rv3066 of Mycobacterium tuberculosis. Nucleic Acids Res. 2012;40(18):9340-55.

15. Engohang-Ndong J, Baillat D, Aumercier M, Bellefontaine F, Besra GS, Locht $C$, et al. EthR, a repressor of the TetR/CamR family implicated in ethionamide resistance in mycobacteria, octamerizes cooperatively on its operator. Mol Microbiol. 2004;51(1):175-88.

16. Ahn SK, Cuthbertson L, Nodwell JR. Genome context as a predictive tool for identifying regulatory targets of the TetR family transcriptional regulators. PLoS One. 2012;7(11):e50562.

17. Bailey $T L$, Boden M, Buske FA, Frith M, Grant CE, Clementi L, et al. MEME SUITE: tools for motif discovery and searching. Nucleic Acids Res. 2009;37(Web Server issue):W202-8.

18. Aravind $L$, Anantharaman $V$, Balaji $S$, Babu MM, Iyer $L M$. The many faces of the helix-turn-helix domain: transcription regulation and beyond. FEMS Microbiol Rev. 2005;29(2):231-62.

19. Mitchell A, Chang HY, Daugherty L, Fraser M, Hunter S, Lopez R, et al. The InterPro protein families database: the classification resource after 15 years. Nucleic Acids Res. 2015;43(Database issue):D213-21.

20. Van der Geize R, Yam K, Heuser T, Wilbrink MH, Hara H, Anderton MC, et al. A gene cluster encoding cholesterol catabolism in a soil actinomycete provides insight into Mycobacterium tuberculosis survival in macrophages. Proc Natl Acad Sci U S A. 2007:104(6):1947-52.

21. Weigoldt M, Meens J, Bange FC, Pich A, Gerlach GF, Goethe R. Metabolic adaptation of Mycobacterium avium subsp. paratuberculosis to the gut environment. Microbiology. 2013;159(Pt 2):380-91.

22. Golby P, Hatch KA, Bacon J, Cooney R, Riley P, Allnutt J, et al. Comparative transcriptomics reveals key gene expression differences between the human and bovine pathogens of the Mycobacterium tuberculosis complex. Microbiology. 2007;153(Pt 10):3323-36.

23. Dutta NK, Mehra S, Kaushal D. A Mycobacterium tuberculosis sigma factor network responds to cell-envelope damage by the promising antimycobacterial thioridazine. PLoS One. 2010;5(4), e10069.

24. Behr MA, Wilson MA, Gill WP, Salamon H, Schoolnik GK, Rane S, et al. Comparative genomics of BCG vaccines by whole-genome DNA microarray. Science. 1999:284(5419):1520-3.

25. Brosch R, Gordon SV, Marmiesse M, Brodin P, Buchrieser C, Eiglmeier K, et al. A new evolutionary scenario for the Mycobacterium tuberculosis complex. Proc Natl Acad Sci U S A. 2002;99(6):3684-9.

26. Galvao TC, Lima CR, Gomes LH, Pagani TD, Ferreira MA, Goncalves AS, et al. The BCG Moreau RD16 deletion inactivates a repressor reshaping transcription of an adjacent gene. Tuberculosis. 2014;94(1):26-33.

27. de la Paz Santangelo M, Klepp L, Nunez-Garcia J, Blanco FC, Soria M, GarciaPelayo MC, et al. Mce3R, a TetR-type transcriptional repressor, controls the expression of a regulon involved in lipid metabolism in Mycobacterium tuberculosis. Microbiology. 2009;155(Pt 7):2245-55.

28. Cuthbertson L, Ahn SK, Nodwell JR. Deglycosylation as a mechanism of inducible antibiotic resistance revealed using a global relational tree for one-component regulators. Chem Biol. 2013;20(2):232-40.

29. Vindal V, Suma K, Ranjan A. GntR family of regulators in Mycobacterium smegmatis: a sequence and structure based characterization. BMC Genomics. 2007;8:289.

30. Ibarra JA, Perez-Rueda E, Segovia L, Puente JL. The DNA-binding domain as a functional indicator: the case of the AraC/XylS family of transcription factors. Genetica. 2008;133(1):65-76.

31. Yu Z, Reichheld SE, Savchenko A, Parkinson J, Davidson AR. A comprehensive analysis of structural and sequence conservation in the TetR family transcriptional regulators. J Mol Biol. 2010;400(4):847-64.

32. Cole C, Barber JD, Barton GJ. The Jpred 3 secondary structure prediction server. Nucleic Acids Res. 2008;36(Web Server issue):W197-201.

33. Nesbitt NM, Yang X, Fontan P, Kolesnikova I, Smith I, Sampson NS, et al. A thiolase of Mycobacterium tuberculosis is required for virulence and production of androstenedione and androstadienedione from cholesterol. Infect Immun. 2010;78(1):275-82.

34. Dover LG, Corsino PE, Daniels IR, Cocklin SL, Tatituri V, Besra GS, et al. Crystal structure of the TetR/CamR family repressor Mycobacterium tuberculosis EthR implicated in ethionamide resistance. J Mol Biol. 2004;340(5):1095-105.

35. Frenois $F$, Baulard AR, Villeret $V$. Insights into mechanisms of induction and ligands recognition in the transcriptional repressor EthR from Mycobacterium tuberculosis. Tuberculosis. 2006;86(2):110-4.
36. Frenois F, Engohang-Ndong J, Locht C, Baulard AR, Villeret V. Structure of EthR in a ligand bound conformation reveals therapeutic perspectives against tuberculosis. Mol Cell. 2004;16(2):301-7.

37. McGuire AM, Weiner B, Park ST, Wapinski I, Raman S, Dolganov G, et al Comparative analysis of Mycobacterium and related Actinomycetes yields insight into the evolution of Mycobacterium tuberculosis pathogenesis. BMC Genomics. 2012;13:120.

38. Galagan JE, Sisk P, Stolte C, Weiner B, Koehrsen M, Wymore F, et al. TB database 2010: overview and update. Tuberculosis. 2010;90(4):225-35.

39. Rutherford K, Parkhill J, Crook J, Horsnell T, Rice P, Rajandream MA, et al. Artemis: sequence visualization and annotation. Bioinformatics. 2000;16(10):944-5

40. Moller S, Croning MD, Apweiler R. Evaluation of methods for the prediction of membrane spanning regions. Bioinformatics. 2001;17(7):646-53.

41. Thompson JD, Higgins DG, Gibson TJ. CLUSTAL W: improving the sensitivity of progressive multiple sequence alignment through sequence weighting, position-specific gap penalties and weight matrix choice. Nucleic Acids Res. 1994;22(22):4673-80.

\section{Submit your next manuscript to BioMed Central and take full advantage of:}

- Convenient online submission

- Thorough peer review

- No space constraints or color figure charges

- Immediate publication on acceptance

- Inclusion in PubMed, CAS, Scopus and Google Scholar

- Research which is freely available for redistribution 